\title{
Retour d'expérience sur Covisan : un dispositif médicosocial pour casser les chaînes de transmission de la Covid-19
}

\section{Sharing of Experience about COVISAN: a Medical and Social System to Break the Sequence of Transmission of COVID-19}

\author{
J. Pernet · H. de Bonnières $\cdot$ C. Breton $\cdot$ V. Hirsch $\cdot$ J.S. Molitor $\cdot$ D. Boutolleau $\cdot$ R. Piarroux $\cdot$ P. Hausfater \\ Reçu le 16 juillet 2020 ; accepté le 4 août 2020 \\ (C) SFMU et Lavoisier SAS 2020
}

Résumé Covisan a été mis en place à partir du 14 avril 2020 au niveau de quatre sites pilotes de l'Assistance publiqueHôpitaux de Paris (APHP) pour casser les chaînes de trans-

\section{J. Pernet $(\bowtie) \cdot$ P. Hausfater}

Service d'accueil des urgences, hôpital Pitié-Salpêtrière, Assistance publique-Hôpitaux de Paris (APHP),

83, boulevard de 1'Hôpital, F-75013 Paris, France

e-mail : julie.pernet@aphp.fr

J. Pernet $\cdot$ C. Breton

Coordination cellule Covisan Pitié-Salpêtrière, hôpital Pitié-Salpêtrière, APHP,

83, boulevard de l'Hôpital, F-75013 Paris, France

H. de Bonnières

Direction des affaires et ressources humaines médicales, hôpital Pitié-Salpêtrière, APHP,

83, boulevard de 1'Hôpital, F-75013 Paris, France

\section{Hirsch}

Direction générale,

Assistance publique-Hôpitaux de Paris, APHP,

3, avenue Victoria, F-75004 Paris, France

J.S. Molitor

Solidarité internationale en Haïti,

siège social, F-92110 Clichy, France

D. Boutolleau

Service de virologie, hôpital Pitié-Salpêtrière, APHP,

83, boulevard de 1'Hôpital, F-75013 Paris, France

D. Boutolleau · R. Piarroux

Institut Pierre-Louis d'épidémiologie et de santé publique,

Inserm, Sorbonne Université, F-75013 Paris, France

R. Piarroux

Service de parasitologie-mycologie,

hôpital Pitié-Salpêtrière, APHP,

Sorbonne Université, F-75013 Paris, France

P. Hausfater

GRC BIOFAST, Sorbonne Université,

91, boulevard de 1'Hôpital, F-75013 Paris, France mission au SARS-CoV-2 selon un modèle original déjà éprouvé en Haïti pour éliminer le choléra dans les années 2010. Le dispositif consiste en un dépistage systématique des cas possibles de Covid-19, un accompagnement dans leur confinement et une prise en charge de leurs proches. Des équipes mobiles se sont déplacées au domicile des cas contacts afin d'évaluer les possibilités d'un isolement au domicile, de proposer des aides matérielles (courses, blanchisserie, hébergement externalisé) et de dépister leurs proches. Au 17 juin 2020, 6376 patients ont été orientés vers Covisan, parmi lesquels 153 avaient une RT-PCR (reverse transciptase polymerase chain reaction) positive au SARSCoV-2. Covisan a permis un partenariat ville-hôpital innovant, en impliquant de multiples acteurs (personnels soignants, administratifs, logisticiens, métiers de service). Les autorités sanitaires se sont d'ailleurs inspirées de ce modèle pour lutter contre l'épidémie en mettant en place le contact tracing. Covisan, qui a appris en marchant, a également rencontré quelques difficultés, en particulier au niveau de la gestion des différents statuts des personnels ainsi qu'au niveau de la communication interne et externe.

Mots clés Dépistage · Chaînes de transmission · Covid-19 Contact tracing $\cdot$ Équipes mobiles

Abstract COVISAN was set up from April 14, 2020 at 4 pilot sites of Assistance publique-Hôpitaux de Paris (APHP) to break the sequence of transmission of SARS$\mathrm{CoV}-2$ according to an original model already proven in Haiti to eradicate cholera in the 2010s. This device relies on a systematic screening of the possible COVID-19 cases, assistance in their containment and care for their close relatives. Mobile teams carried out home visits to evaluate the possibilities of home confinement, to propose material help (errands, laundry, outsourcing accommodation) and to propose a screening of their relatives. By June 17, 2020, 6376 patients have been moved towards COVISAN, and 
153 had a positive RT-PCR to SARS-CoV-2. COVISAN has made possible an innovating town-hospital partnership, involving multiple actors (nursing staff, administrative staff, logistician, and service professions). Health authorities have decided to model their strategy to control the SARSCoV-2 spread closely to COVISAN's, and implemented "contact tracing". COVISAN that learned by doing, has also encountered some difficulties, mainly concerning the different staff statutes and also with the internal and external communication.

Keywords Screening - Sequence of transmission · COVID$19 \cdot$ Contact tracing $\cdot$ Mobile teams

\section{Introduction}

Covisan a été mis en place pour casser les chaînes de transmission du SARS-CoV-2, optimiser les mesures d'isolement des personnes contaminées, limiter l'apparition de microfoyers, favoriser l'acceptation du confinement avec des mesures d'accompagnement et affiner la surveillance épidémiologique. Ce dispositif est inspiré de celui mis en place en Haïti pour l'élimination du choléra [1-2], survenu en octobre 2010 [3]. Sur ce modèle, Covisan s'est déployé sur la région parisienne, a permis un dépistage systématique des cas possibles de Covid-19, proposé un accompagnement au confinement des patients et une prise en charge de l'entourage - le contact tracing — par le biais d'équipes mobiles, réalisant des visites à domicile dans les foyers suspects.

\section{Historique : une démarche inspirée d'une expérience concluante en Haïti}

Le projet Covisan est directement inspiré de l'expérience d'élimination du choléra en Haïti dans les années 2010. En octobre 2010, neuf mois après un important tremblement de terre, le choléra a été accidentellement importé en Haïti [3]. Cette épidémie, considérée comme la plus importante des dernières décennies a été responsable de plus de 800000 cas et 9791 décès d'après le ministère de la Santé haïtien [4]. L'origine de l'épidémie a pu être identifiée au niveau d'un camp de casques bleus népalais situé en amont $\mathrm{du}$ fleuve Artibonite, dans la commune de Mirebalais qui déversait ses fosses septiques dans ce cours d'eau [5], provoquant aussitôt une épidémie qui, en quelques semaines, s'est propagée à l'ensemble du territoire haïtien [6].

À partir de juillet 2013, le gouvernement haïtien, l'Unicef et d'autres partenaires internationaux ont lancé un plan national d'élimination du choléra (2013-2022). Il a été mis en œuvre selon un modèle original s'appuyant sur des équipes mobiles d'intervention rapide (EMIRA) intervenant sur des zones d'intervention ciblées en fonction des données épidémiologiques $[1,7,8]$. Ces EMIRAs intervenaient sur tout cas suspect de choléra et avaient pour mission de protéger l'entourage des patients, par l'apprentissage des mesures d'hygiène, la chloration de surfaces potentiellement contaminées (en particulier les latrines), la distribution de savon et de pastilles de chlore pour la décontamination de l'eau. Les équipes étaient le plus souvent accompagnées d'infirmiers chargés de dépister des cas dans le voisinage et d'administrer un traitement antibiotique (doxycycline ou azythromycine, selon l'âge) aux sujets contacts. Fin 2016, le renforcement de cette stratégie dans les suites du cyclone Matthew s'est accompagné d'une chute drastique de la transmission du choléra, et depuis février 2019, plus aucun nouveau cas de choléra n'a été diagnostiqué en Haïti.

Comme pour le choléra, après le pic épidémique de Covid-19, des équipes mobiles ont été déployées en Îlede-France, afin d'aider les patients à protéger leurs proches. Il s'agit de dépister les patients suspects d'infection à la Covid-19 et de stopper la propagation de la maladie en optimisant les conditions d'isolement des malades.

\section{Mise en place du dispositif Covisan}

Le dispositif Covisan a été mis en place à partir du 14 avril 2020 sous l'égide des pouvoirs publics (mission nationale déconfinement, préfecture de région, Agence régionale de santé (ARS) Île-de-France, Assistance publique-Hôpitaux de Paris (APHP) au niveau de quatre sites pilotes : les hôpitaux Avicenne, Louis-Mourier, Bichat (porté par la Communauté professionnelle territoriale de santé [CPTS] du $18^{\mathrm{e}}$ ) et Pitié-Salpêtrière.

Covisan est constitué d'une équipe projet installée sur le campus Picpus de l'APHP composée de membres de l'APHP et de bénévoles, et initialement de quatre sites pilotes qui se sont transformés en antennes Covisan et qui ont été rejoints par d'autres structures de santé, en collaboration avec de nombreux partenaires d'intervention (hébergement, blanchisserie, nettoyage, portage de repas/livraisons de courses, supervision médicale et soignante dans les lieux d'isolement).

Covisan est un dispositif largement partenarial. Il regroupe :

- différentes institutions : des hôpitaux, des organisations de médecine de ville, des centres de santé, des collectivités territoriales, des organisations non gouvernementales (ONG), des associations, des hôtels, des entreprises de service. L'Union régionale des professionnels de santé (URPS) médecins libéraux de l'Île-de-France, la ville de Paris, la région Île-de- France, la mairie de Saint-Ouen, le département Seine-Saint-Denis, la Croix-Rouge française, la mairie d'Aubervilliers, la mairie de Bondy, la 
protection civile, l'APHP, Médecins Sans Frontière, le Groupe Accor ont entre autres permis la mise en place de Covisan ;

- différentes professions : des professionnels de santé (médecins, étudiants en médecine, infirmiers, pharmaciens, biologistes), des intervenants sociaux, des logisticiens, des métiers de service ;

- des acteurs publics, privés et bénévoles.

Le but du dispositif est de casser les chaînes de transmission du SARS-CoV-2 et d'accompagner la levée du confinement (11 mai 2020) en proposant un dépistage systématique aux patients potentiellement infectés par le SARS-CoV-2 ainsi qu'à leurs proches et de les accompagner dans leur confinement. Les patients pouvant se confiner efficacement au domicile ont bénéficié d'un "kit patient » constitué d'équipements de protection individuelle (masques chirurgicaux, solution hydroalcoolique) et d'explications orales et écrites concernant les gestes barrières et si besoin de solutions d'accompagnement (blanchisserie, courses, repas, portage de médicaments). Les patients ne pouvant s'isoler de façon efficace à leur domicile se sont vu proposer un hébergement hors du domicile (hôtel ou centre d'hébergement), sur la base du volontariat et ont bénéficié d'un suivi pour lever leur isolement.

Les patients sont rentrés dans le dispositif soit via les services des urgences (SU), les médecins généralistes, les centres de dépistage, les Samu/Smur, l'application COVIDOM, soit via les différents services d'hospitalisation et de consultation des hôpitaux. Le flux de patients a été géré en sectorisant le rayon d'action des différentes antennes Covisan (Fig. 1). Les patients se trouvant dans une zone non couverte par une antenne étaient pris en charge par l'équipe de Picpus.

\section{Covisan Pitié-Salpêtrière, devenu Covisan 13- 14-15}

Le pilote de la Pitié-Salpêtrière a été ouvert le 14 avril 2020. Son fonctionnement était assuré à ses débuts par la direction de l'hôpital, un cadre de l'APHP, un encadrement de proximité assuré par des membres de la protection civile, un médecin coordonnateur des urgences, des médecins hospitaliers volontaires pour assurer les consultations et initialement sept bénévoles ayant un profil social, logistique et des étudiants ou internes de médecine et pharmacie pour réaliser les prélèvements nasopharyngés pour les RT-PCR. Le centre accueillait les patients sept jours sur sept de $10 \mathrm{~h}$ à $20 \mathrm{~h}$. Cette antenne était localisée au sein de l'hôpital de la PitiéSalpêtrière à Paris jusqu'au 7 juin 2020 et a ensuite déménagé le 8 juin dans des locaux de la mairie du $14^{\mathrm{e}}$ arrondissement de Paris et est désormais cordonné par les CPTS des $13^{\mathrm{e}}, 14^{\mathrm{e}}$ et $15^{\mathrm{e}}$ arrondissements de Paris (centre Covisan 1314-15). Au 10 juin 2020, 63 bénévoles (dont du personnel navigant d'Air France), quatre assistantes sociales de

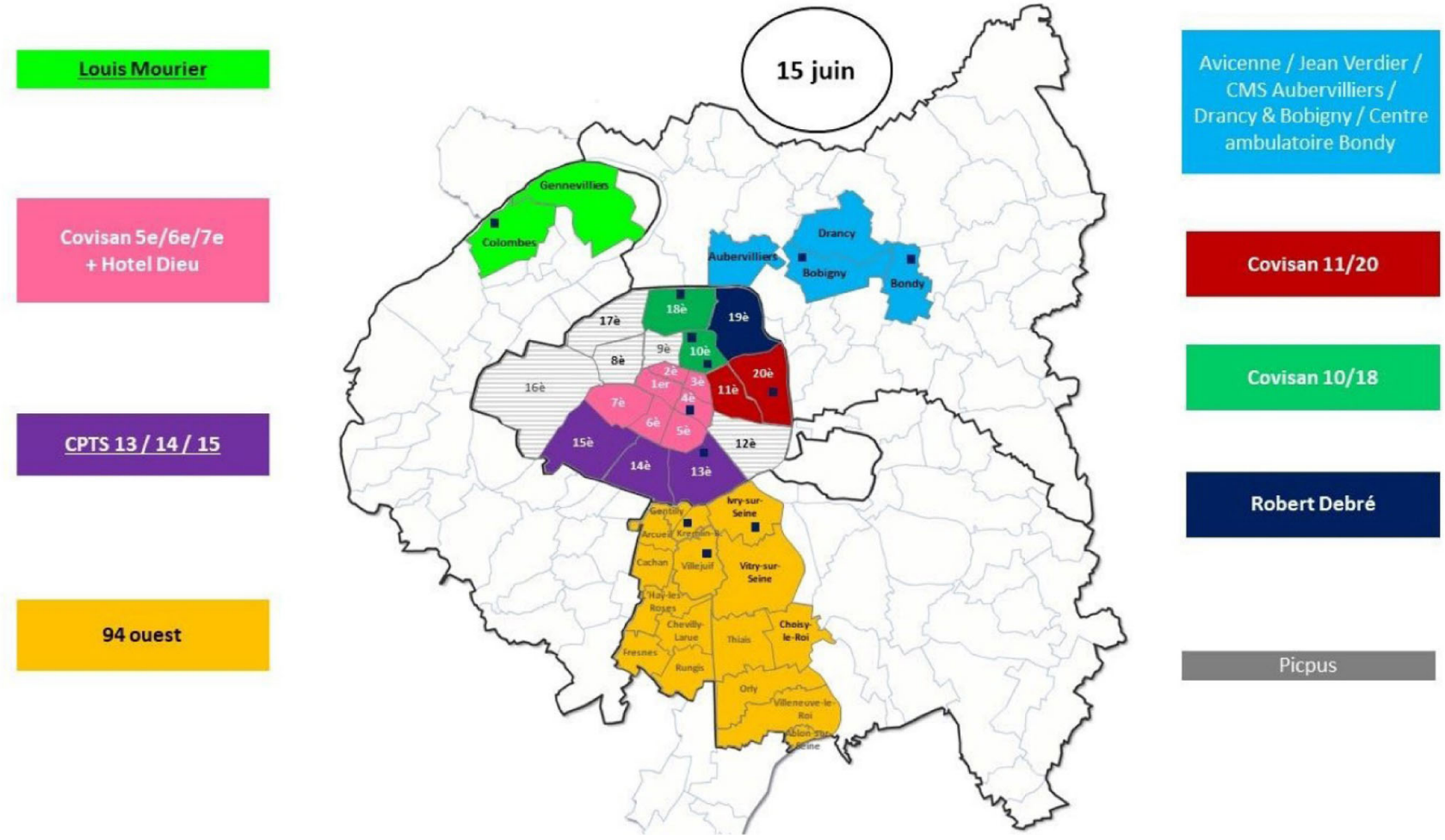

Fig. 1 Cartographie des centres Covisan au 15 juin 2020. Document fourni par le centre Picpus, Assistance publique-Hôpitaux de Paris 


\begin{tabular}{|c|c|c|}
\hline Suspicion de Covid-19 forte & $\begin{array}{l}\text { Suspicion de Covid modérée : } \\
\text { plusieurs symptômes non } \\
\text { spécifiques associés }(\geq 2)\end{array}$ & $\begin{array}{l}\text { Suspicion de Covid faible : } \\
\text { symptôme non spécifique } \\
\text { associé isolé }\end{array}$ \\
\hline Anosmie/agueusie isolées & Toux & Toux \\
\hline $\begin{array}{l}\text { Syndrome grippal avec fièvre, courbatures, frissons depuis } \\
\text { moins d'une semaine }\end{array}$ & Rhinorrhée & Rhinorrhée \\
\hline Dyspnée avec polypnée et toux & Céphalées & Céphalées \\
\hline Lésions cutanées évocatrices & Diarrhées & Diarrhées \\
\hline $\begin{array}{l}\text { Douleur thoracique à type de sensation de gêne } \\
\text { médiothoracique en barre avec dyspnée }\end{array}$ & Fièvre & Fièvre \\
\hline \multicolumn{3}{|l|}{$\begin{array}{l}\text { Diarrhées et douleurs abdominales chez un patient de plus } \\
\text { de } 70 \text { ans }\end{array}$} \\
\hline $\begin{array}{l}\text { Symptômes grippaux non typiques mais contact rapproché } \\
\text { avec patients diagnostiqués Covid+ dans les } 72 \text { heures }\end{array}$ & & \\
\hline
\end{tabular}

l'hôpital et huit infirmières intérimaires ont participé au fonctionnement du centre.

Les patients du centre Covisan 13-14-15 étaient adressés par les médecins généralistes des $13^{\mathrm{e}}, 14^{\mathrm{e}}$ et $15^{\mathrm{e}}$ arrondissements de Paris, le SU, les services de consultation ou hôpitaux de jour de l'hôpital de la Pitié-Salpêtrière, s'ils étaient considérés comme potentiellement infectés par le SARSCoV-2 sur le questionnaire d'entrée préconsultation. Les patients adressés par les urgences de l'hôpital Saint-Joseph et les patients hospitalisés pour infection à la Covid-19 dans les différents services de l'hôpital de la Pitié-Salpêtrière ont aussi été inclus dans le dispositif.

Le patient entrant dans le dispositif Covisan bénéficiait tout d'abord d'une évaluation médicale qui classait le cas en faiblement, moyennement ou fortement suspect [9-11] (Tableau 1). Une RT-PCR nasopharyngée à la recherche du SARS-CoV-2 a été proposée systématiquement au patient, qui a toujours été acceptée. Au décours, il a été proposé un entretien social (Annexe $\mathrm{A}$ ) visant à évaluer ses conditions de vie. Cette étape permettait d'établir la constitution du foyer, d'apprécier les caractéristiques du logement, avec possibilités ou non d'isoler le patient de ses proches, de savoir si le patient a des personnes à charge (enfants, personnes dépendantes) et d'évaluer ses besoins en cas de nécessité de confinement. À cette occasion, un temps était consacré à l'explication et à la démonstration des mesures barrières. Il était ensuite proposé au patient d'organiser une visite à domicile pour dépister ses proches et vérifier la faisabilité de l'isolement. Au cours de cette visite, des « kits » étaient fournis, comprenant des masques chirurgicaux et des flacons de solution hydroalcoolique.

Une fois la situation évaluée et sans attendre les résultats de RT-PCR, un plan d'action était anticipé pour le patient qui était ou non confirmé en fonction des symptômes des membres du foyer et de leurs résultats de RT-PCR. Par exemple si, dans un foyer constitué de quatre personnes, une personne est symptomatique (quels que soient les résultats de RTPCR) et que les autres personnes sont asymptomatiques mais que, parmi elles, une a une RT-PCR positive, l'isolement se fait en général au domicile du patient alors qu'on aurait pu penser initialement proposer un hébergement externalisé au cas index. Ces décisions se faisaient en concertation avec le médecin présent au centre et l'équipe mobile ayant pris en charge le patient.

La cellule Covisan Pitié-Salpêtrière a permis d'identifier quelques microclusters, en particulier les patients s'étant rendus à des enterrements ou à des rassemblements religieux. Quelques personnes vivant en foyer se sont aussi spontanément présentées à la consultation, ce qui a permis d'alerter l'ARS de leur situation et d'organiser des dépistages de masse au niveau de leur structure de vie. Au 10 juin 2020 , la cellule Covisan 13-14-15 avait organisé 300 visites (et 596 RT-PCR) à domicile, 417 RT-PCR dans ses locaux, soit 1013 prélèvements.

Rapidement, cette antenne Covisan a pris l'initiative de suivre les patients ayant une PCR positive pour le SARS$\mathrm{CoV}-2$, les patients fortement suspects cliniquement mais avec RT-PCR négative, ainsi que leurs contacts asymptomatiques, pour s'assurer de la bonne évolution des symptômes pour les uns et l'absence d'apparition de symptômes pour les autres. À ce jour, aucun cas contact n'a secondairement déclaré de symptômes compatibles avec une infection Covid-19.

\section{Premiers résultats, évolution et devenir de Covisan}

Le tableau 2 résume les chiffres clés de l'ensemble des antennes Covisan et ceux de Covisan 13-14-15 au 19 mai 2020 et au 17 juin 2020. Ces chiffres ont été fournis par le 
siège de Covisan à Picpus, de façon quotidienne. Le choix du 19 mai a été retenu, car se situant à mi-chemin entre la mise en place du dispositif et la rédaction de cet article. On constate une nette augmentation des cas contacts entre les deux dates : au 19 mai, un cas index permettait d'identifier 1,3 contact, alors qu'au 17 juin il permettait d'en identifier 1,8 . On observe par ailleurs une diminution du taux de RTPCR positives, ce qui est en adéquation avec les chiffres nationaux [12].

Au 30 juin, 1158 patients ont été suivis par Covisan 1314-15, parmi lesquels 1102 (95\%) ont été prélevés par les équipes Covisan. Parmi les RT-PCR réalisées, 63 (6\%) sont revenues positives. Sept cent quarante-six (64\%) patients ont été vus à domicile, $412(36 \%)$ sur site.
Les places d'hôtels ont été fermées le 9 juin, faute de patients à héberger, et la fermeture de Chardon-Lagache (structure d'hébergement pour patients en grande précarité, située au sein de l'hôpital Chardon-Lagache, APHP) a eu lieu le 22 juin 2020.

$\mathrm{Au} 15$ juin 2020, les différentes équipes Covisan ont été approvisionnées en tests rapides d'orientation diagnostique (TROD) Covid-19 en appliquant les recommandations de la Haute Autorité de santé (HAS) [13]. En cas de TROD positif, une RT-PCR était systématiquement proposée ainsi qu'un test sérologique en laboratoire de biologie médicale.

Au 17 juin 2020, l'activité était en baisse dans les différentes antennes, et il a donc été proposé de regrouper les centres et d'organiser des équipes d'astreinte pour la réalisation des visites à domicile (Fig. 2).

Tableau 2 Activité de Covisan et Covisan 13-14-15

\begin{tabular}{|lllll|}
\hline & 19 mai 2020 & & 17 juin 2020 & Covisan 13-14-15 \\
\hline & Covisan (total) & Covisan 13-14-15 & Covisan (total) & 547 \\
Cas index & 1346 & 414 & 2245 & 462 \\
Cas contacts & 1694 & 286 & 4131 & 225 \\
Visite à domicile & 660 & 150 & 1510 & $500 / 29(6 \%)$ \\
RT-PCR à domicile/ & $1369 / 84(6 \%)$ & $318 / 21(7 \%)$ & $3393 / 153(4 \%)$ & 15 \\
positive & 146 & 9 & 157 & 27 \\
Livraisons de courses/ & & & 101 \\
repas & 78 & 24 & & \\
Hébergement hors & & & \\
domicile & & & & \\
\end{tabular}

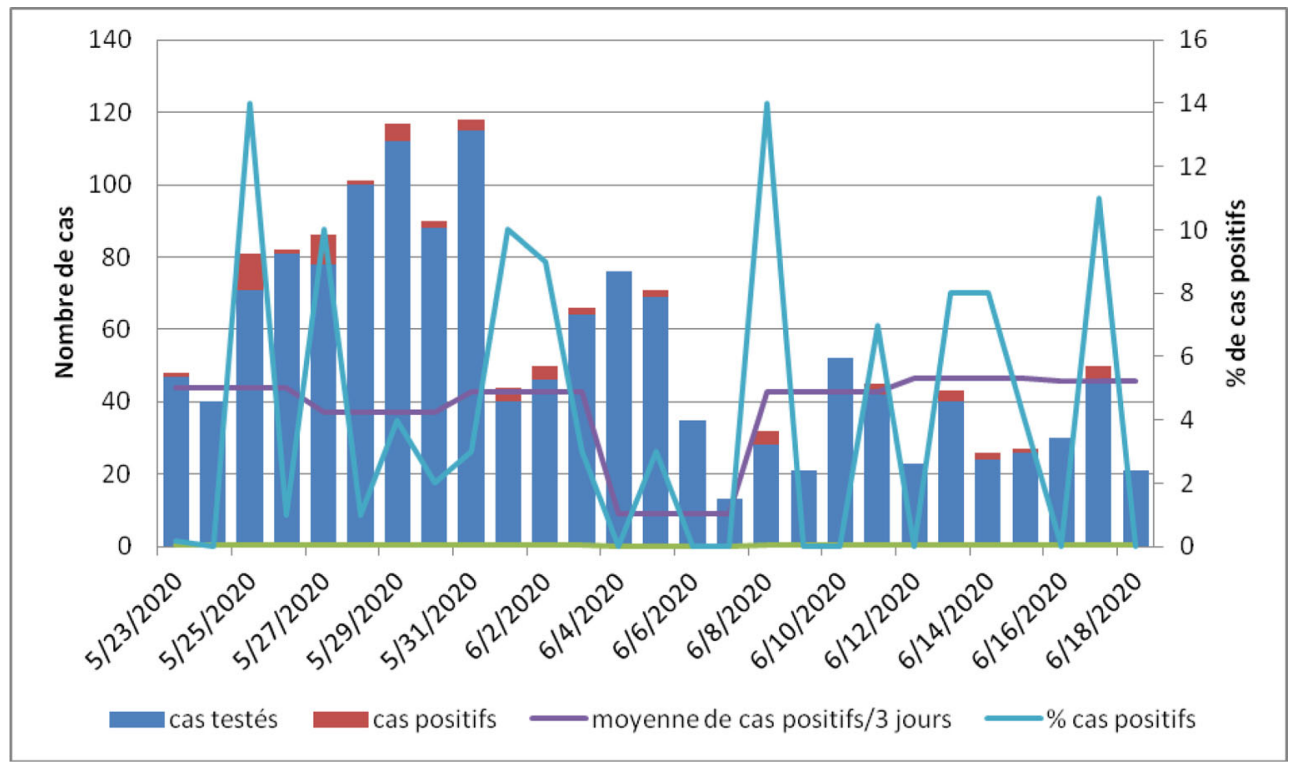

Fig. 2 Nombre de RT-PCR SARS-CoV-2 réalisées à Covisan du 23 mai 2020 au 18 juin 2020 et taux de positivité. Cellule Picpus de l'Assistance publique-Hôpitaux de Paris. RT-PCR : Reverse Transcriptase Polymerase Chain Reaction 


\section{Discussion}

Un des grands succès de Covisan a été l'adhésion des patients inclus dans le dispositif : au 30 juin, 1158 patients ont été suivis par Covisan 13-14-15, parmi lesquels 1102 (95\%) ont été prélevés par le centre : les patients non prélevés avaient dans la majorité des cas déjà été prélevés ailleurs. Les quelques refus se sont essentiellement portés sur les enfants. Une telle adhésion a pu être obtenue grâce à la dimension humaine du dispositif reposant sur les équipes d'intervention initialement constituées d'un binôme incluant un acteur social et un acteur de soins puis en général de deux acteurs de soins, qui ont tissé un lien de confiance très fort avec les patients en fournissant des kits de protection individuelle, en prenant le temps d'expliquer les gestes barrières ainsi qu'en proposant des conseils personnalisés sur leur isolement. Ces kits étaient constitués de masques chirurgicaux et de solutés hydroalcooliques, dont le nombre était adapté à la composition du foyer. Outre le geste, ces kits ont été très bien accueillis par les familles, car ils ont été distribués à un moment où il était difficile de se les procurer, étant en rupture de stock. Peu d'hébergements externalisés ont été proposés, car très souvent, il était possible d'organiser un isolement à domicile efficace. Quand ils étaient proposés, ils ont dans la quasi-totalité été acceptés, et ce en l'absence de coercition. Cette adhésion a aussi été favorisée par le dispositif en lui-même qui prenait en charge le patient dans sa globalité, en plaçant le patient au centre du dispositif, aussi bien sur le plan médical que social via les nombreuses aides proposées (courses, blanchisserie, etc.).

Par certains côtés, on peut considérer que Covisan a constitué un exemple puisque, à partir du 11 mai 2020, les autorités sanitaires ont décidé de mettre en place un dispositif similaire pour faire face à l'épidémie. Ce contact tracing, mis en place par la Caisse primaire d'assurance maladie (CPAM), consiste à rechercher des cas contacts pour les patients ayant une RT-PCR positive, donnée transmise directement par les laboratoires à la CPAM, via SI-DEP (Système d'informations de dépistage), une plateforme sécurisée où sont systématiquement enregistrés les résultats des tests Covid-19 des laboratoires. Un téléservice dénommé « contact Covid » élaboré par l'assurance maladie et accessible via Amelipro permet d'enregistrer l'ensemble des informations concernant le cas index et les éventuels cas contacts recensés par le médecin généraliste mais aussi par Covisan, via les résultats RT-PCR directement transmis. Dans le cas d'un prélèvement positif, une plateforme de niveau 2 est opérée par des équipes de l'assurance maladie qui est chargée de finaliser la recherche de cas contacts. Elle prend contact dans les 24 heures avec chacun des cas contacts afin de les inviter à rester confinés à domicile, à réaliser un test diagnostique dans un délai dépendant de la date du contact avec le cas index et de proposer des mesures d'accompagnement au cours de l'isolement. Ce dispositif présente donc de nombreux points communs avec Covisan. Cependant, il a été considéré que son action n'était pas redondante, car il intervient uniquement en cas de résultat de RT-PCR positif et avec un délai de mise en place plus long, dû au temps nécessaire pour pratiquer le test et rendre les résultats. D'après la littérature, la sensibilité de la RT-PCR peut être estimée entre 56 et $83 \%$ [14-16], ce qui justifie de prendre en compte aussi la probabilité clinique et la mise en place des mesures d'isolement malgré un résultat négatif. De plus sont inclus dans Covisan des patients qui auraient pu échapper au dispositif national mis en place par l'assurance maladie, en particulier les patients migrants, ou vivant en foyer, ou n'ayant pas de médecin traitant. Covisan a réussi à inclure dans son dispositif un certain nombre de patients précaires, qui se sont présentés spontanément au niveau des SU. Grâce à son implantation parfois hospitalière, Covisan a pu bénéficier de services de traduction et de l'aide d'assistantes sociales pour favoriser la compréhension et l'adhésion au dispositif. L'intervention d'acteurs locaux a aussi favorisé l'adhésion du dispositif dans certains quartiers, qui n'aurait pas été possible via le système impersonnel et à distance proposé par la CPAM. Du fait de ce succès, une mission est en cours pour déployer un programme similaire à Covisan en Guyane où la situation est aujourd'hui préoccupante.

La démarche Covisan a aussi permis d'identifier des foyers épidémiques (au sein de foyers de migrants ou de jeunes travailleurs, d'écoles) ou des situations présentant un risque de propagation de l'épidémie (enterrement, répétition de pièce de théâtre) et d'initier des actions de maîtrise de l'épidémie à plus grande échelle. Cette action est habituellement du ressort de l'ARS, mais dans ce cas, il est arrivé que Covisan prenne les devants et organise des dépistages de masse dans ces structures ou que l'ARS s'appuie sur les équipes de Covisan pour mener différentes opérations. Le dispositif Covisan a permis de créer un lien très fort entre la médecine de ville et l'hôpital, en particulier avec les SU : dès le début, les médecins de ville ont été impliqués dans les décisions opérationnelles et stratégiques du dispositif, et depuis le transfert de Covisan Pitié-Salpêtrière à la ville, la direction de l'hôpital et les médecins hospitaliers restent engagés dans le projet.

Une des difficultés rencontrées par Covisan a été la communication de la mise en place du dispositif, tant au niveau hospitalier qu'en ville, mais aussi entre le siège de Covisan à Picpus et les différentes antennes Covisan. Initialement, des points de situation quotidiens par téléconférence ont été mis en place avec toutes les antennes, qui ont été espacés à deux par semaine. Ces points de situation mêlaient l'opérationnel et la stratégie, et n'ont fait que tardivement ( 8 juin) l'objet d'ordres du jour et de comptes rendus écrits. Si un projet similaire devait voir le jour, il parait essentiel de prévoir 
dès sa mise en place une communication structurée, claire et protocolisée.

Les relations avec l'ARS ont été initialement compliquées pour l'intervention dans les foyers ou les clusters identifiés par Covisan. Ces missions étant habituellement dans les prérogatives de l'ARS, Covisan l'alertait en vue d'une intervention rapide ; devant son absence de réactivité, à plusieurs reprises, Covisan a organisé lui-même ces missions. Progressivement, les relations avec l'ARS se sont améliorées et ont abouti à une collaboration avec en général un appui humain et matériel par Covisan.

Initialement, les ressources humaines de Covisan aussi bien au niveau du siège que des différentes antennes étaient bénévoles, ce qui pendant le confinement a permis à un certain nombre d'individus de se sentir utiles pendant cette crise. Leur présence a permis une humanisation essentielle lors de leurs interventions, particulièrement délicates au domicile des patients, qui a permis une grande adhésion au dispositif, et ce sans aucune coercition. Avec la structuration de Covisan et sa pérennisation, certains d'entre eux ont été contractualisés. Ce mélange d'acteurs bénévoles et contractuels occupant les mêmes fonctions a été source d'incompréhensions et parfois de tensions. À l'avenir, si un projet similaire devait voir le jour, il parait important de ne s'appuyer sur des bénévoles que pour une période limitée dans le temps, peut-être un mois, pour éviter cette situation.

Covisan a été mis en place le 14 avril 2020, soit un mois et demi après le début de l'épidémie en France. La Corée du Sud a mis en place dès le mois de février, donc au début de l'épidémie, un dispositif de contact tracing et de dépistage de masse dans son pays, ce qui lui a permis de lutter efficacement contre la propagation du SARS-CoV-2 $[17,18]$. Cela n'a pas été réalisé en France, ce qui aurait peut-être été utile, mais dans tous les cas, sa mise en pratique aurait été compliquée : la France ne disposait pas alors de tests de dépistage fiables - et une fois disponibles, leur usage était initialement réservé aux patients nécessitant une hospitalisation - ni de masques ou de solutés hydroalcooliques en quantité suffisante.

En cas de deuxième vague, Covisan serait en capacité de faire face : plus de 500 personnels ont été formés au dispositif et au prélèvement nasopharyngé. Il faudrait néanmoins impliquer les partenaires de ville dès le déploiement tout en maintenant les liens avec les différents hôpitaux, définir plus clairement l'articulation avec l'ARS, la CPAM et tout autre acteur institutionnel, planifier les besoins en ressources humaines très en amont, mettre à disposition les outils de gestion unifiés, harmoniser les procédures entre antennes, renforcer le lien entre les différentes antennes et la cellule centrale en projetant régulièrement les ressources de la cellule centrale dans les antennes et structurer la communication partenariale autour du dispositif pour assurer l'inclusion. D'une façon plus générale, on pourrait imaginer que le modèle de Covisan existe sous forme de cellule de veille qui serait activable lors d'une prochaine crise sanitaire. En effet, de par sa structure originale, son mode d'intervention rapide, léger, efficace et humain, ainsi que le nombre de personnels formés, Covisan est un outil précieux à préserver.

\section{Conclusion}

Covisan, un dispositif francilien, a permis rapidement après le pic de l'épidémie de Covid-19 de mettre en place un dépistage d'envergure des cas index ainsi que de leurs contacts et de les accompagner dans leur confinement. Son intérêt a été reconnu, et une partie des activités a été reproduite au niveau national par la CPAM et des ARS. Enfin, il a permis une collaboration ville-hôpital inédite et devrait déboucher sur de nouveaux projets de collaboration.

Liens d'intérêts : les auteurs déclarent ne pas avoir de liens d'intérêts.

\author{
Éléments remarquables \\ - Gestion de projet avec de multiples acteurs \\ - Une réponse efficace face au développement des foyers \\ épidémiques \\ - Création du lien de confiance avec le patient \\ - Partenariat ville-hôpital
}

\section{Pistes d'amélioration}

- Prérogatives et champs d'intervention pas toujours clairs entre Covisan et Agence régionale de santé (ARS)

- Écart dans système rémunération public-privé et mélange des statuts

- Circulation de l'information :

- entre le siège de l'Assistance publique-Hôpitaux de Paris

et les antennes Covisan

- entre les services hospitaliers et les antennes Covisan 
Annexe A. Entretien social utilisé par Covisan 13-14-15 : (Disponible dans le Matériel supplémentaire)

\section{Références}

1. Rebaudet R, Bulit G, Piarroux R, et al (2019) The case-area targeted rapid response strategy to control cholera in Haiti: A fouryear implementation study. PLoS Negl Trop Dis 13:e0007263

2. Piarroux R, Rebaudet S (2019) La surprenante disparition du choléra en Haïti. Rev Prat 69:763-5

3. Piarroux R, Barrais R, Faucher B, et al (2011) Understanding the cholera epidemic, Haiti. Emerg Infect Dis 17:1161-8

4. Republic of Haiti. Ministry of Public Health and Population. Centre de documentation. Bulletins cholera 2010-2018. http:// mspp.gouv.ht/newsite/documentation.php (Dernier accès le 22 juin 2020)

5. Tasker F, Robles F (2010) Source of cholera outbreak may never be known, Miami Herald, November 20, 2010. http://www.miamiherald.com/2010/11/20/1934870/source-of-haitis-cholera-outbreak.html\#ixzz1EKEo8pqB (Dernier accès le 22 juin 2020)

6. Gaudart J, Rebaudet S, Barrais R, et al (2013) Spatio-temporal dynamics of cholera during the first year of the epidemic in Haiti. PLoS Negl Trop Dis 7:e2145.7

7. Finger F, Bertuzzo E, Luquero FJ, et al (2018) The potential impact of case-area targeted interventions in response to cholera outbreaks: a modeling study. PLoS Med 15:e1002509

8. Piarroux R (2019) Choléra, Haïti 2010-2018, histoire d'un désastre. CNRS Éditions, Paris

9. Haut Comité de santé publique (2020) Avis du 5 mars 2020 relatif à la prise en charge des cas confirmés d'infection au virus Sars-CoV-2. Https://www.hcsp.fr/explore.cgi:avisrapportsdomaine?clefr $=771$ (Dernier accès le 22 juin 2020)

10. Haut Comité de santé publique (2020) Avis du 23 mars 2020 relatif aux recommandations thérapeutiques dans la prise en charge du Covid-19 (complémentaire à l'avis du 5 mars 2020).
Https://www.hcsp.fr/explore.cgi:avisrapportsdomaine?clefr=785 (Dernier accès le 22 juin 2020)

11. Haut Comité de santé publique (2020) Avis du 8 avril 2020 relatif à la prise en charge à domicile ou en structure de soins des cas de Covid-19 suspectés ou confirmés. Https://www.hcsp.fr/explore. cgi:avisrapportsdomaine?clefr $=793$ (Dernier accès le 22 juin 2020)

12. Santé publique France (2020) Covid-19. Point épidémiologique hebdomadaire du 18 juin 2020. https://www.santepubliquefrance.fr/maladies-et-traumatismes/maladies-et-infections-respiratoires/infection-a-coronavirus/documents/bulletin-national/covid19-point-epidemiologique-du-18-juin-2020 (Dernier accès le 22 juin 2020)

13. Haute Autorité de santé (2020) Rapport d'évaluation. Place des tests sérologiques rapides (TDR, TROD, autotests) dans la stratégie de prise en charge de la maladie Covid-19. Validé par le Collège le 14 mai 2020. https://www.has-sante.fr/upload/docs/application/pdf/2020-05/rapport_tests_serologiques_rapides_covid19 vd.pdf (Dernier accès le 22 juin 2020)

14. Ai T, Yang Z, Xia L, et al (2020) Correlation of chest CT and RTPCR testing in coronavirus disease 2019 (Covid-19) in China: a report of 1,014 Cases. Radiology 296:E32-E40

15. Fang Y, Zhang H, Ji W, et al (2020) Sensitivity of chest CT for Covid-19: comparison to RT-PCR. Radiology 296:E115-E7

16. Wang W, Xu Y, Tan W, et al (2020) Detection of SARS-CoV-2 in different types of clinical specimens. JAMA 323:1843-4

17. Ferguson N, Laydon D, Nedjati-Gilani G, et al (2020) Impact of non-pharmaceutical interventions (NPIs) to reduce Covid-19 mortality and healthcare demand. https://www.imperial.ac.uk/ media/imperial-college/medicine/sph/ide/gida-fellowships/Imperial-College-COVID19-NPI-modelling-16-03-2020.pdf (Dernier accès le 17 juillet 2020)

18. Osong Public Health and Research Perspectives (2020) Covid-19 National Emergency Response Center, Epidemiology \& Case Management Team, Korea Centers for Disease Control \& Prevention. Contact transmission of Covid-19 in South Korea: novel investigation techniques for tracing contacts 11:60-3 (Dernier accès le 17 juillet 2020) 